\title{
Fluoxetine and Sleep EEG: Effects of a Single Dose, Subchronic Treatment, and Discontinuation in Healthy Subjects
}

\author{
Bernd Feige, Ph.D., Ulrich Voderholzer, M.D., Dieter Riemann, Ph.D., Professor of Clinical \\ Psychophysiology, Ralf Dittmann, M.D., Ph.D., Fritz Hohagen, M.D., Professor of Psychiatry, \\ and Mathias Berger, M.D., Professor of Psychiatry
}

\begin{abstract}
The goals of the current study were to evaluate whether a single dose of fluoxetine causes qualitatively different changes in sleep architecture and NREM sleep EEG than subchronic administration in healthy subjects and to determine degree and duration of such changes after the single dose and after discontinuation from subchronic administration. Our hypothesis was that subchronic intake should cause changes qualitatively different from the single dose and that such changes could be sufficiently long-lived to suggest the possibility of a dosing in intervals of several days. Ten healthy volunteers first took one single 60-mg dose of fluoxetine and a week later started to take a 40-mg dose every morning for three weeks. Sleep laboratory nights included two nights before and four nights after the single dose and every second night for two weeks after discontinuation from subchronic administration. The single dose caused only a slight increase in drug plasma concentrations but relatively clear changes in sleep structure. After discontinuation from subchronic administration, sleep quality indices normalized quickly
\end{abstract}

(within 2-4 days), whereas REM latency and spectral power effects correlated with total SSRI plasma concentration and normalized more slowly, corresponding to the drug plasma half-life of about 10 days. The REM fraction of the sleep period showed a rebound, whereas the delta sleep ratio did not correlate with drug plasma levels and yet remained increased after the medication interval. Thus, the only qualitative difference seen between acute and subchronic medication was the initial sleep disturbance. REM latency and especially the delta sleep ratio remained increased for several days after discontinuation from subchronic administration, indicating the possibility of a less-than-daily maintenance medication after an initial daily interval. Finally, the pattern of change observed for the delta sleep ratio indicates that it may be due to secondary, adaptive effects possibly linked to the antidepressant effect of fluoxetine in depressed patients.

[Neuropsychopharmacology 26:246-258, 2002] (C) 2002 American College of Neuropsychopharmacology. Published by Elsevier Science Inc.
From the Department of Psychiatry and Psychotherapy, AlbertLudwigs-University, Freiburg, Germany (BF, UV, DR, FH, MB); and Lilly Deutschland GmbH and Psychosomatic Department, Children's Hospital, University of Hamburg, Hamburg, Germany (RD).

Address correspondence to: Dr. Bernd Feige, Department of Psychiatry and Psychotherapy, Albert-Ludwigs-University Freiburg, Hauptstraße 5, 79104 Freiburg, Germany.

Received 22 September 2000; revised 30 May 2001; accepted 6 July 2001.

Online publication: $7 / 10 / 01$ at www.acnp.org/citations/ Npp071001148.
KEY WORDS: Human; Sleep; EEG spectral effects; Antidepressants; SSRIs

In the last years, selective serotonin reuptake inhibitors (SSRIs) have increasingly replaced tricyclics in antidepressive treatment due to their more benign side effect profile. Still, compliance in long-term maintenance therapy often enough is insufficient, since many patients are reluctant to accept daily dosing of antidepressants and thus tend to omit medication sometimes. It is well known for most antidepressants that a discontinu- 
ation for some days can lead to relapse into depression (for a review, cf. Viguera et al. 1998).

The SSRIs currently available are mainly distinguished by their plasma halflife, which is particularly long for fluoxetine (1-3 days after single and 4-6 days after chronic administration) and its active metabolite norfluoxetine (7-14 days) (Hyttel 1994). Therefore, the question arises what the time course of central nervous effects of a single and a subchronic dose of fluoxetine is: If the effects should last for several days after discontinuation, this would be an indication of a higher level of security of this medication with respect to compliance problems. It has already been suggested that the long plasma half-life is the reason for the negligible adverse effects reported upon fluoxetine discontinuation (Zajecka et al. 1998; Lejoyeux and Adés 1997; Haddad 1997; for a recent definition of a SSRI discontinuation syndrome cf. Schatzberg et al. 1997).

Furthermore, there are several indications that important central nervous effects of antidepressant medication are of a secondary, adaptational nature, prompting a comparison of the time course of these effects with plasma drug concentration. Especially the fact that several clearly distinct pharmacological interventions all result in an amelioration of depressive symptoms in responders, and do so only with a delay of some days to weeks, indicates that the actual antidepressant effect and change in symptomatology is an indirect consequence of the drug action. A delay of about two weeks has even been used to discriminate true drug effects from spontaneous remissions in pattern analytical assessments of antidepressive efficacy (Quitkin et al. 1984, 1987), although the delayed onset hypothesis has been challenged in favor of a triggering or 'kick-starting' concept (Stassen and Angst 1998). Shortened time to remission using pulse loading schemes has also been reported (down to about $72 \mathrm{~h}$ by bolus injection of imipramine; Malhotra and Santosh 1996). Finally, a previous study found a significant antidepressive effect of a two-day treatment with the tricyclic antidepressant clomipramine (plasma half-life of about 21 hours) 5 days after either oral or intravenous dosing (Pollock et al. 1989; Kupfer et al. 1989). These findings indicate that a relatively short-term plasma presence of antidepressants might be sufficient in order to initiate the necessary neurophysiological changes leading to an amelioration of depressive symptoms.

In order to measure central nervous effects of fluoxetine, we performed a spectral analysis of the sleep EEG in healthy human volunteers. Specific spectral EEG effects of antidepressants have been reported by several authors (Tarn et al. 1993; Saletu and Grünberger 1985; Patat et al. 1998; Fischer et al. 1986; Yamadera et al. 1987; Saletu et al. 1991; Röschke et al. 1997); furthermore, there is evidence for a link between sleep regula- tion and depression, with sleep homeostasis being routinely characterized by spectral EEG parameters (Borbély et al. 1988; Achermann et al. 1993).

Evidence for a link between sleep structure and depression comes from the fact that sleep deprivation (which actually constitutes a manipulation of sleep homeostasis) is an effective, albeit often short-lived, antidepressant treatment in about $70 \%$ of patients with major depressive disorder (Riemann et al. 1999; Berger et al. 1997; Vollmann and Berger 1993). Furthermore, there are consistent findings of a REM sleep disinhibition (shortened REM latency, higher REM density; cf. Vogel 1983; Kupfer et al. 1976; Berger and Riemann 1993) and a NREM delta power shift towards the second NREM episode (decreased delta sleep ratio; Kupfer et al. 1990; Buysse et al. 1997) as correlates of depression. A decreased delta ratio has also been found to increase the likelihood of further depressive episodes in the absence of pharmacotherapy (Spanier et al. 1996).

Most antidepressants normalize both of these parameters in depressed patients, i.e., they increase REM latency (Vogel 1983) and delta ratio (Kupfer et al. 1994). The increase in delta ratio has been found to be associated with clinical response to clomipramine (Ehlers et al. 1996). An increase in REM latency has been observed in healthy subjects after administration of fluoxetine (Vasar et al. 1994). This indicates that sleep EEG changes in normals can be used as a model of the antidepressant effect of a substance.

It should be noted, however, that some clinically effective antidepressants do not suppress REM sleep, e.g., trimipramine (Vogel et al. 1990; Sonntag et al. 1996; Wiegand and Berger 1989) and the postsynaptic serotonin antagonist and presynaptic serotonin and norepinephrine reuptake inhibitor nefazodone (Vogel et al. 1998; Armitage et al. 1997; Rush et al. 1998; Gillin et al. 1997). Thus, REM latency changes may be associated with the mechanism of action of many antidepressants as previously suggested (Vogel 1983; Vogel et al. 1975; this includes many SSRIs and specifically fluoxetine; $\mathrm{cf}$. Buysse et al. 1999; Trivedi et al. 1999), but not of all antidepressants. Much less is known about delta ratio changes due to various antidepressants, allowing the speculation that the delta sleep ratio could be closer to a general mechanism of antidepressant action than REM latency.

In the current study we therefore examined sleep architecture and NREM sleep EEG changes in the course of a single dose and subsequent subchronic application of fluoxetine for signs of secondary, adaptive changes and the time course of normalization after discontinuation for implications regarding clinical application schemes. Our hypothesis was that subchronic intake should cause qualitatively different changes than the single dose in one of the parameters linked to depression, i.e., REM latency or delta sleep ratio. These 
changes could furthermore be sufficiently long-lived to suggest the possibility of a fluoxetine dosing in intervals of several days for antidepressive maintenance therapy (e.g. once-weekly; cf. Schmidt et al. 2000). The study was conducted with healthy male subjects instead of depressed patients in order to reduce possible confounding effects of an amelioration of the depressive state.

\section{MATERIALS AND METHODS}

Ten healthy male volunteers aged between 20 and 34 years $(27.8 \pm 4.1$ years) with a body weight between 60 and $90 \mathrm{~kg}(71.9 \pm 7.5 \mathrm{~kg})$ were enrolled. There were no abnormal findings upon clinical and laboratory examinations in any of the subjects. None of them had a personal or family history of psychiatric disorders, allergies, adverse drug reactions, or major surgical interventions involving the gastrointestinal tract. Participants were drug free, had no history of drug or alcohol abuse, and none of then had participated in other drug studies within a month preceding the start of this trial. Written consent was obtained from each subject. The experiments were performed in accordance to the Helsinki Declaration of 1975. The protocol was approved by the Ethical Committee of the University of Freiburg.

Capsules containing $20 \mathrm{mg}$ of fluoxetine and matching placebo capsules were used. The single dose consisted of three capsules $(60 \mathrm{mg})$ and the daily dose in the subchronic phase was two capsules ( $40 \mathrm{mg}$ ).

\section{Study Design}

On the first study day, inclusion/exclusion criteria were evaluated, including demographic data and medical history. A full physical examination, including blood pressure, pulse and weight measurements, EEG (12 leads), neurological, and psychiatric examinations were performed. Laboratory tests included a full blood count (including platelets), clinical chemistry: sodium, potassium, chloride, calcium, urea, serum creatinine, glucose, total protein, albumin, total bilirubin, SGOT, SGPT, AP, GGT, LDH, HbsAg and HIV, T3, T4, TSH, and urine analysis (dip stick): urobilinogen, glucose, $\mathrm{pH}$, protein, nitrite and blood.

Study nights 2 through 4 and every second night from night 28 to 42 (cf. the study design overview, Figure 1) included standard sleep recordings of horizontal EOG, submental EMG and EEG (C3-A2; C4-A1). Time in bed was held constant from 23:00 to 7:00 h; subjects were required to adhere to this fixed sleep schedule and to remain abstinent from alcohol during the whole course of the study. The polysomnograms were scored blindly in epochs of $30 \mathrm{~s}$ by two experienced raters according to standardised criteria (Rechtschaffen and Kales 1968). Blood samples drawn at 22:00 h before each study night were centrifuged and frozen on site and sent to an external laboratory (Simbec Research Ltd., UK) for measurement of fluoxetine and norfluoxetine plasma concentrations by gas chromatography. For statistical analysis, values below the detection threshold were set to the detection threshold (1.957 ng/ $\mathrm{ml}$ for fluoxetine and $2.239 \mathrm{ng} / \mathrm{ml}$ for norfluoxetine). During the three-week medication interval, additional blood samples were drawn on nights 14 and 21 and analyzed for plasma concentrations to ensure compliance.

\section{Conventional Sleep Measures}

Polysomnographical parameters derived from visual staging were total sleep time, the fraction of each sleep (or wake) stage within the sleep period (SP) in percent, REM latency, and the number of eye movements in REM sleep. Sleep onset was defined as the first occurrence of stages 2, 3, 4, or REM sleep (one 30-s epoch was sufficient). The sleep period ended correspondingly with the first wake or stage 1 epoch not followed by deeper sleep until the end of bedtime. Sleep latency is the time between the start of bed time (lights out) and sleep onset. Total sleep time is the time within the sleep period not spent awake. Sleep efficiency is defined as the percentage of total sleep time within bedtime. REM

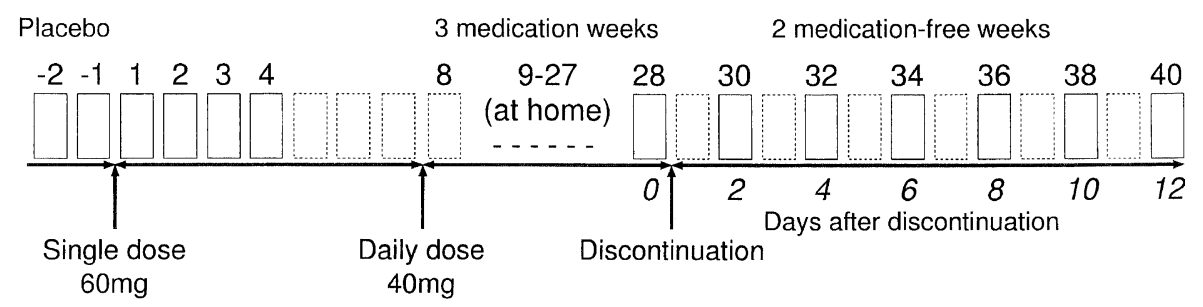

Figure 1. Study design. Nights spent in the sleep lab are shown as boxes with solid lines, nights spent at home with broken lines. In the morning prior to the baseline night $(-1)$, subjects received placebo capsules; in the morning before night 1 , they received a single dose of $60 \mathrm{mg}(3 \times 20 \mathrm{mg})$ fluoxetine, and in each morning preceding nights $8-28$ they received a dose of $40 \mathrm{mg}(2 \times 20 \mathrm{mg})$. 
latency is the time between sleep onset and the first occurrence of REM sleep.

For "REM latency-wake," epochs scored as wake that may have occurred between sleep onset and REM sleep were excluded. REM density, finally, was calculated as the number of REM eye movements per 30-s epoch multiplied by 10 . This can be interpreted as a percentage where a REM density of $100 \%$ is defined as 10 eye movements per $30 \mathrm{~s}$ ( 20 per minute).

\section{Spectral Analysis}

During the night, continuous EEG (C3 referenced to the right ear) was amplified with a time constant of $0.3 \mathrm{~s}$ and a lowpass at $70 \mathrm{~Hz}$, digitized at $102.4 \mathrm{~Hz}$ and stored for off-line analysis. Due to the sampling theorem, this led to an aliasing of frequencies above $51.2 \mathrm{~Hz}$ into the frequencies between 32.4 and $51.2 \mathrm{~Hz}$, which therefore were excluded from the analysis of sleep spectral power changes (but used in the artifact detection algorithm).

An all-night spectral analysis was performed on the same 30-s epochs for which sleep stages had been determined. Within each 30-s epoch, spectral power was calculated using the Fast Fourier Transform (FFT) algorithm from forty-six 128-point windows overlapping by half, resulting in a spectral resolution of $0.8 \mathrm{~Hz}$. Within each FFT window separately, the EEG was demeaned and detrended by subtracting the linear least-squares regression line before applying a Welch window and calculating the FFT. The 46 spectral power estimates were averaged in order to increase the stability of the estimate. The estimates obtained for each frequency and 30-s epoch were continuously stored on disk.

The goal of the further analysis was to minimize the effects of confounding variables on the spectra averaged across epochs, such as the number of movements or arousals and other sleep parameters that can be analyzed separately (cf. Feige et al. 1999). This was done by excluding 'deviant' epochs from the average. Deviant epochs were those containing movements or arousals as determined during staging; furthermore, the total (0.8-48 $\mathrm{Hz}$ ) and gamma-band (32-48 Hz) log power of each epoch was related to the corresponding median-filtered value (the median of values in the $5 \mathrm{~min}$ preceding and 5 min following the epoch), and an epoch was excluded if the deviation was larger than the difference between the median and the first quartile of all median-filtered values across the night. In this way, artifacts mainly restricted to low frequencies (such as EOG events) as well as those occurring mainly in higher frequencies (such as EMG contamination) were eliminated in a data-driven way.

\section{Statistical Analysis}

The logarithmic (base $e$ ) spectra for artifact-free NREM sleep epochs were averaged across each night and addi- tionally (in order to calculate the delta sleep ratio) within each NREM sleep episode. Logarithmic spectral band power was calculated after adding the spectral power values within the following frequency bands: Delta $0.1-3.5 \mathrm{~Hz}$, theta $3.5-8 \mathrm{~Hz}$, alpha $8-12 \mathrm{~Hz}$, sigma $12-16 \mathrm{~Hz}$, beta1 $16-24 \mathrm{~Hz}$, and beta $24-32 \mathrm{~Hz}$. The delta sleep ratio is the quotient of mean delta band power values in the first NREM sleep episode to the corresponding mean in the second NREM sleep episode (Kupfer et al. 1990).

From the average log spectral power values, the corresponding average for the baseline night was subtracted for each subject in order to derive baseline-relative spectra due to the properties of the logarithm.

The significance of baseline-relative sleep/spectral parameter changes throughout the experiment was assessed using a repeated measures ANOVA with the within-subject factor NIGHT. Two-sided contrasts were used within these ANOVAs to assess the statistical significance of the baseline deviation for each night. Correlations between plasma concentrations and sleep/spectral parameters were evaluated by ANOVA with the (individually baseline-subtracted) parameter entered as variate and plasma level entered as covariate.

\section{Pharmacodynamic Modeling of Spectral Power Effects}

It is not within the scope of the current study to derive full pharmacokinetic / pharmacodynamic models for all observed sleep and spectral parameters. We will focus upon the pharmacodynamic relationship between drug plasma concentrations $C$ and $\log$ spectral power values $E$, for which an established and simple model, the $E_{\max }$ model, is available (cf. Bellissant et al. 1998):

$$
E=E_{0}+E_{\max } \cdot \frac{C^{\gamma}}{\left(C E_{50}\right)^{\gamma}+C^{\gamma}}
$$

where $E_{0}$ (power at concentration 0$), E_{\max }$ (maximum power change), $C E_{50}$ (concentration at half the maximum effect), and $\gamma$ (Hill coefficient) are the model parameters. Sigma, beta1 and beta2 frequency bands were analyzed in parallel; $C E_{50}$ and $\gamma$ were modeled globally across subjects and frequency bands, whereas $E_{0}$ and $E_{\max }$ were allowed to be different for each subject and frequency band. Nonlinear least-squares optimization was performed using the 'nls' package of S-PLUS (MathSoft, Cambridge, MA).

\section{RESULTS}

The upper left panel of Figure 2 shows the plasma concentrations of fluoxetine and its active metabolite norfluoxetine. After the single $60 \mathrm{mg}$ dose, plasma levels increased to $31 \pm 30 \mathrm{ng} / \mathrm{ml}$ (mean $\pm \mathrm{SD}$ ) for fluoxetine 

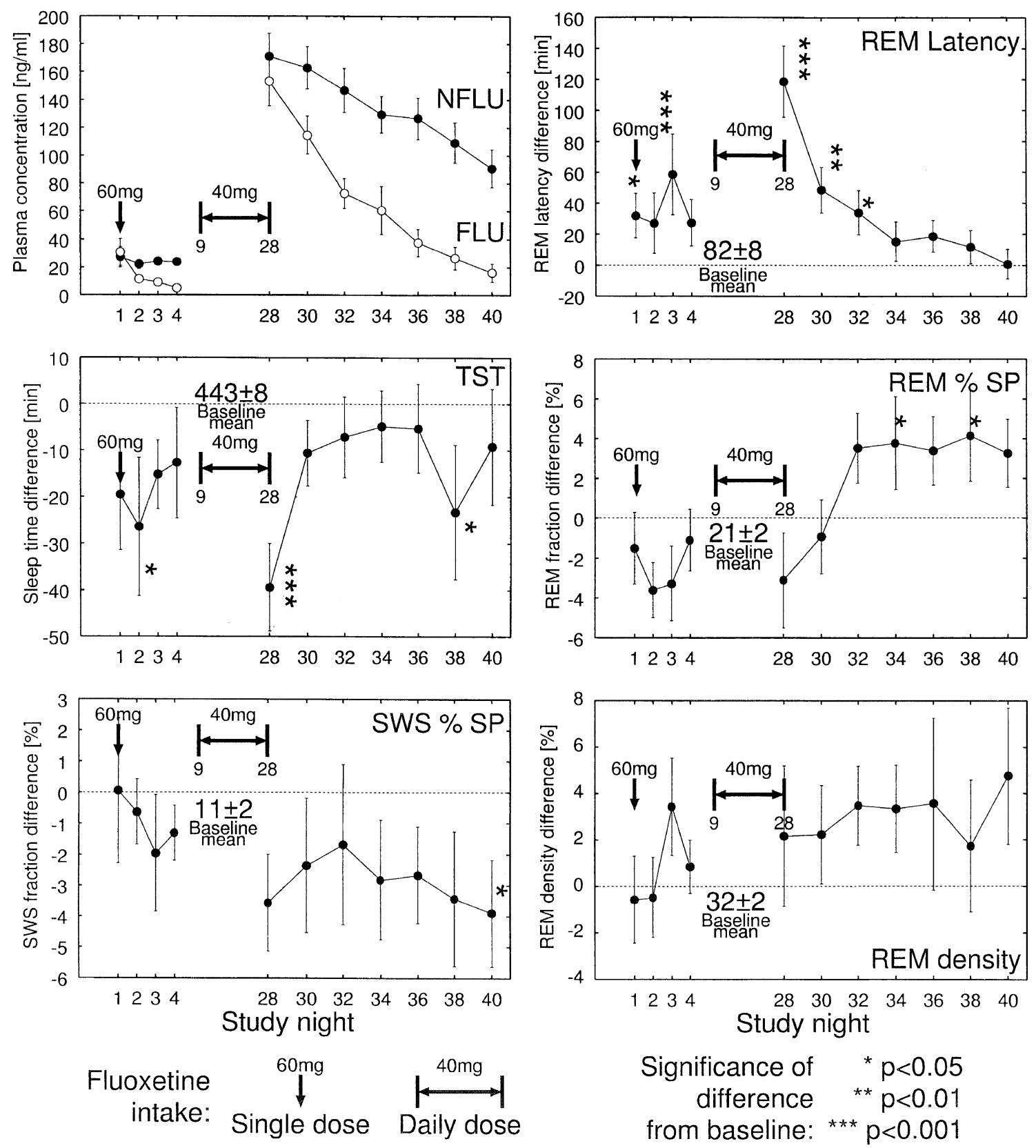

Figure 2. Study time courses of fluoxetine (open circles) and norfluoxetine (closed circles) plasma concentrations (top left panel) and of the baseline-subtracted sleep parameters: Total sleep time (TST), slow-wave sleep fraction of sleep period (SWS \% SP), REM latency, REM fraction of sleep period, and REM density. The baseline levels (mean $\pm S E$ ) are noted above or below the dotted zero (baseline) axis within each graph. Note that TST is significantly decreased at the end of the subchronic medication period but recovers quickly, while REM latency is significantly increased and takes longer to normalize. While REM density is not changed significantly, the REM sleep fraction is acutely suppressed but increased after discontinuation. At most one missing value was allowed in any single average $(9 \leq N \leq 10)$. Significance levels for 2 -sided contrasts against baseline are indicated.

and $27 \pm 23 \mathrm{ng} / \mathrm{ml}$ for norfluoxetine, while reaching $153 \pm 56 \mathrm{ng} / \mathrm{ml}$ and $171 \pm 53 \mathrm{ng} / \mathrm{ml}$ on day 28 , after three weeks of the daily $40 \mathrm{mg}$ dose. The large variability is in line with previous reports of large interindividual differences in plasma concentration following oral intake of a fixed dose (DeVane 1992).
REM latency and total sleep time (TST) were already changed significantly after the single dose and normalized within six and two days, respectively, after discontinuation from subchronic intake. The REM fraction of the sleep period showed a rebound four days after discontinuation. 
Figure 3 shows the averaged baseline-relative spectral power for the nights after three weeks of medication (night 28) and subsequent discontinuation. The baseline-relative drug effect is a clear spectral power increase, starting at about $12 \mathrm{~Hz}$, with a peak in the sigma band (around $14 \mathrm{~Hz}$ ) and, with rising tendency, up to the highest observed frequency $(32 \mathrm{~Hz})$. After discontinuation, the spectral increment generally decayed with time after discontinuation but did not reach the baseline level at the end of the observed time interval, 12 days after discontinuation. The beta-range increment (16-32 Hz) did not decrease monotonically but peaked around night 34 , causing spectral power on this night to be the second largest after night 28 .

The time course of spectral changes of NREM sleep EEG (slight suppression of delta wave activity, increase in sigma- and beta-band activity; cf. Figure 4) was generally similar to that of fluoxetine and norfluoxetine plasma concentrations (as shown in the upper left panel of Figure 2): they were not significant after the single dose but marked after the subchronic intake, remaining significant between 8 and 12 days after discontinuation.

While the total delta power across nights was changed only weakly by the drug (about $10 \%$ reduction at the end of the subchronic phase; ANOVA $p=.031$; cf. Table 1), the delta ratio was increased, i.e., the distribution of delta power across NREM episodes was clearly shifted towards the first episode (cf. Figure 4, upper right panel). A single dose caused a slight but not significant increase in delta ratio. In the last medication night (night 28), a significant delta ratio increase could be seen on the $5 \%$ level despite a large variability. Between two and four days after discontinuation (nights 30-32), the difference to baseline did not reach significance but subsequently increased and remained signifi- cant up to the last observed night (12 days after discontinuation).

Table 1 summarizes the effects of fluoxetine on polysomnographic and spectral parameters. Changes are divided into three categories: nights with significant effects after a single dose, duration of persistence after discontinuation from subchronic medication, and delay of rebound effects after this discontinuation, determined by 2 -sided contrasts against baseline $(p<.05)$. Sleep quality-related parameters such as sleep efficiency and fraction of stage 2 sleep showed a relatively early response and low persistence; REM fraction and REM total eye movements showed a rebound. Spectral EEG parameters were only weakly influenced by the single dose, but showed long persistence after discontinuation from subchronic dosing and no rebound effects during the time of observation.

One well-known source of variance in clinical trials with fluoxetine is due to the large interindividual differences in the pharmacokinetics of this substance and the correspondingly large differences in plasma levels within a fixed intake scheme. For this reason, we also examined the correlations between plasma concentrations of fluoxetine, norfluoxetine, and their sum, which approximately represents the amount of SSRI present in the plasma since the two substances are nearly equipotent as SSRIs. All three concentrations were examined in order to increase the specificity of the analysis. Since, for example, the norfluoxetine concentration decreased about linearly after discontinuation, any effect which also evolves in one direction in this time interval will correlate with the norfluoxetine concentration, without necessarily indicating a causal link. If, however, the sum of the two concentrations with its initially more rapid decrease (due to the fluoxetine component) corre-

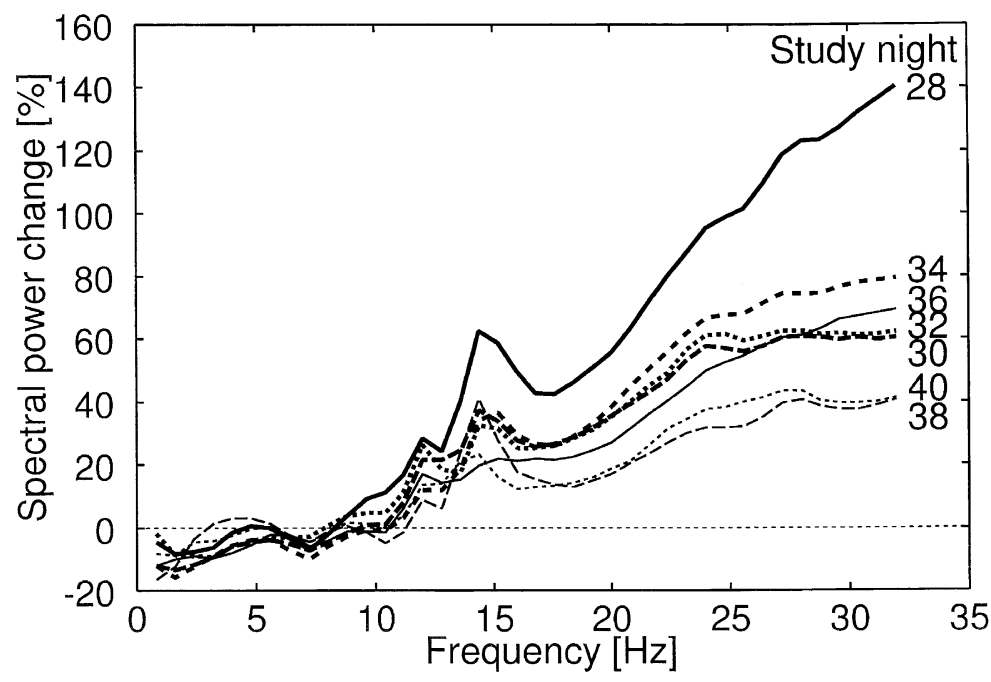

Figure 3. Grand-average baseline-relative NREM EEG spectra for the study nights following the subchronic medication interval, displayed as the spectral power difference in percent relative to the baseline night. 
Table 1. Influence of Fluoxetine/Norfluoxetine on sleep: Changes Relative to Baseline; ANOVA and Baseline Contrast Results

\begin{tabular}{|c|c|c|c|c|}
\hline & $\begin{array}{c}\text { Significance of } \\
\text { Factor NIGHT, } \\
\text { ANOVA }\end{array}$ & $\begin{array}{c}\text { Study Nights } \\
\text { with Significant } \\
\text { Effects after Single } \\
\text { Dose }(p<.05)\end{array}$ & $\begin{array}{c}\text { Persistence of } \\
\text { Significant Effects } \\
\text { (Duration in Days after } \\
\text { Discontinuation; } p<.05 \text { ) }\end{array}$ & $\begin{array}{c}\text { Presence of Significant } \\
\text { Rebound Effects (Single } \\
\text { Nights after } \\
\text { Discontinuation; } p<.05 \text { ) }\end{array}$ \\
\hline Sleep latency & 0,349 & - & - & - \\
\hline Total sleep time & 0,002 & $2(\downarrow)$ & $0(\downarrow)$ & - \\
\hline Sleep efficiency & 0,002 & $2(\downarrow)$ & $0(\downarrow)$ & - \\
\hline Wake \% SP & 0,000 & - & $2(\uparrow)$ & - \\
\hline Stage $1 \% \mathrm{SP}$ & 0,276 & - & - & - \\
\hline Stage $2 \%$ SP & 0,032 & $3(\uparrow)$ & - & - \\
\hline SWS \% SP & 0,071 & - & - & - \\
\hline REM \% SP & 0,005 & - & - & $6,10(\uparrow)$ \\
\hline REM latency & 0,000 & $1,3(\uparrow)$ & $4(\uparrow)$ & - \\
\hline REM lat.-wake & 0,000 & $3(\uparrow)$ & $4(\uparrow)$ & - \\
\hline REM tot.eye mts. & 0,001 & $2(\downarrow)$ & - & $4-8,12(\uparrow)$ \\
\hline REM density & 0,220 & - & - & - \\
\hline Delta & 0,031 & - & - & - \\
\hline Theta & 0,580 & - & - & - \\
\hline Alpha & 0,858 & - & - & - \\
\hline Sigma & 0,000 & - & $12(\uparrow)$ & - \\
\hline Beta1 & 0,000 & - & $8(\uparrow)$ & - \\
\hline Beta2 & 0,000 & - & $8(\uparrow)$ & - \\
\hline Delta ratio & 0,000 & - & $12(\uparrow)$ & - \\
\hline
\end{tabular}

Direction of change relative to baseline: $\uparrow$ increase, $\downarrow$ decrease.

Significance and characteristics of changes for sleep parameters and NREM sleep EEG spectral values. Parameters with significant NIGHT effect appear in bold print. The three columns following the ANOVA significance characterize the changes by listing the nights at which a significant change occurred after the single dose, the duration of persistence after discontinuation ( 0 ' meaning that there only was a significant effect in the night following the last dose, night 28), and by listing the nights after discontinuation at which rebound effects occurred. The arrow after each number indicates the direction of change relative to baseline. Significance of a change for a given night was evaluated using 2-sided contrasts against baseline, $p<0.05$.

lates more strongly than any single concentration, this observation is a stronger indication towards a modification of the observed parameter by the momentary SSRI plasma level. Furthermore, the acute and discontinuation phases were analyzed separately in order to discriminate effects of brief and longer-term exposure.

Figure 5 shows scatterplots visualizing the correlations with plasma concentration for two of the variables: REM latency (left two columns) and sigma-band NREM EEG spectral power (right two columns). In order to remind the reader how plasma concentrations evolved in a particular phase of the experiments, 'time arrows' have been inserted in the plots. After the single intake, plasma levels first rose, then fell, whereas they steadily decreased after discontinuation from subchronic intake. For both REM latency and sigma-band spectral power, a clear correlation with the (slowly decreasing) norfluoxetine and summed SSRI concentrations can be observed for the time after subchronic medication and discontinuation. These parameters also scatter across large ranges in the acute dosing phase but without clear correlation to the plasma concentrations. This indicates that the initial dose of $60 \mathrm{mg}$ causes relatively strong, interindividually different sleep changes, whereas the slow diminution of plasma concentration after discontinuation leads to a correspondingly slow normalization of these sleep parameters.

Table 2 summarizes the plasma concentration correlations found for all observed parameters. REM latency and spectral power effects correlated best with total SSRI concentration, but only in the time interval after discontinuation. On the other hand, the correlations with the sleep stage 2 decrease and wake increase were largest after the single dose and with the fluoxetine concentration, indicating an initial perturbation with quick recovery.

The spectral power correlations were similar across all frequency bands above $10 \mathrm{~Hz}$ and strongest in the sigma and beta 1 bands, indicating a single functional origin of these spectral changes. The sigma band showed a negative correlation with fluoxetine and a tendential $(p=.07)$ positive correlation with norfluoxetine after the single dose, indicating an onset of sigma band changes together with norfluoxetine buildup (i.e., with a time delay of about one day) after the single dose. Finally, while the delta ratio was clearly changed by the medication (cf. Figure 4), this change did not correlate with any drug plasma concentration.

Since spectral power values correlated most strongly with the sum of fluoxetine and norfluoxetine concentrations and showed no signs indicating the development 

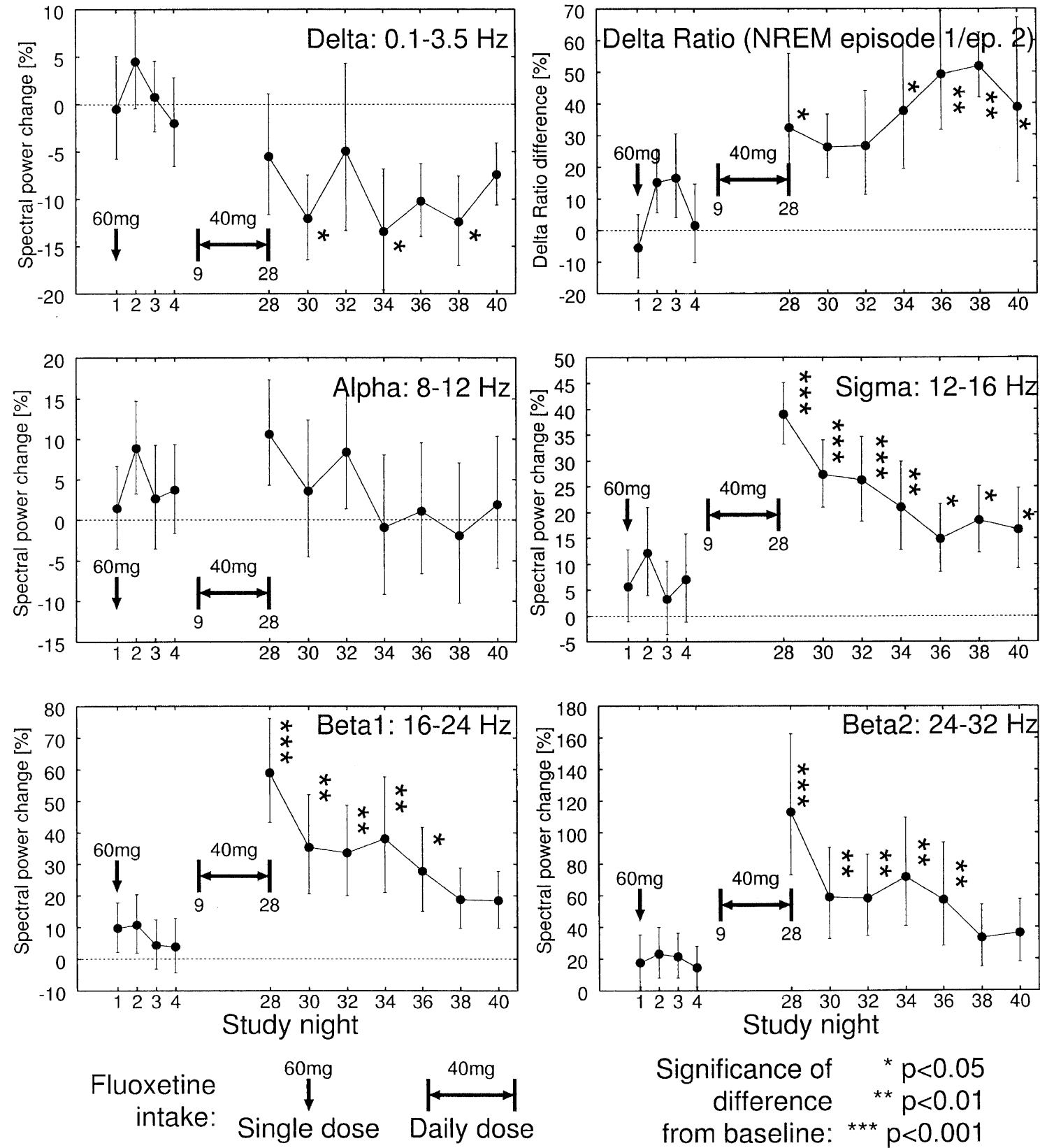

Figure 4. Study time courses of the changes in NREM EEG spectral power, measured as the difference in percent relative to the baseline night, for different frequency bands. In addition, the top right graph shows the change in delta ratio relative to the baseline.

of tolerance, it can be assumed that a simple $E_{\max }$ model is applicable for these effects. Furthermore, sigma, beta1, and beta2 bands showed similar and strong correlations with total SSRI plasma concentration and were therefore analyzed within a single joint optimization model (see Methods). The global parameters were modeled as (estimate $\pm S E) C E_{50}=204 \pm 57 \mathrm{ng} / \mathrm{ml}$ and $\gamma=1.3 \pm 0.6$. The parameters modeled individually for each subject and frequency band, $E_{0}$ and $E_{\max }$, were (mean $\left.\pm S D\right) 2.1 \pm 0.3$ and $0.4 \pm 0.3$ for sigma, $0.6 \pm 0.2$ and $0.7 \pm 0.5$ for beta1, and $-0.3 \pm 0.2$ and $1.0 \pm 0.9$ for beta 2 .

\section{DISCUSSION}

In this study, fluoxetine induced four different types of dynamic changes in the sleep EEG:

- Sleep quality measures (sleep efficiency, total sleep time) showed a clear worsening upon the initial dose but quick recovery (within one or two days) after both the single dose and discontinuation from subchronic administration despite the long plasma halflife of 1-2 weeks; 


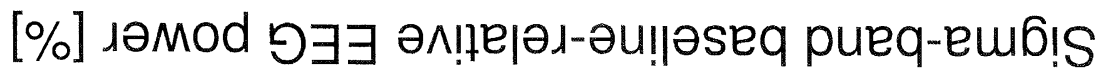

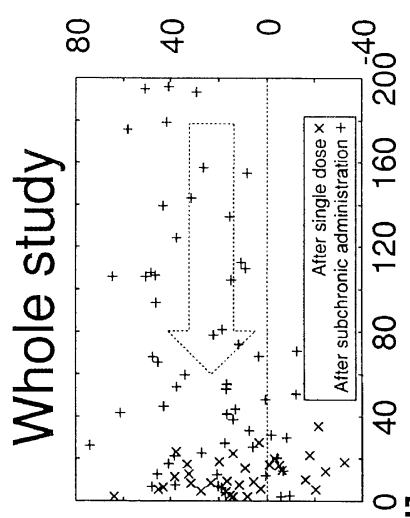

$\infty$ o $\circ$ i

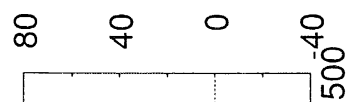

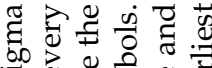

के वे है है च

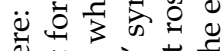

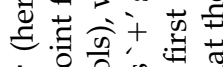

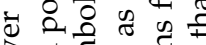

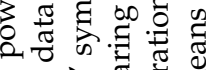

๘

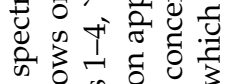

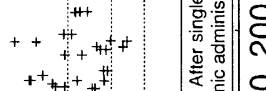

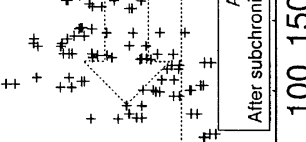

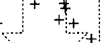

$8=$

का की
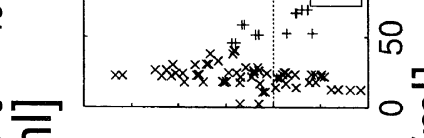

음
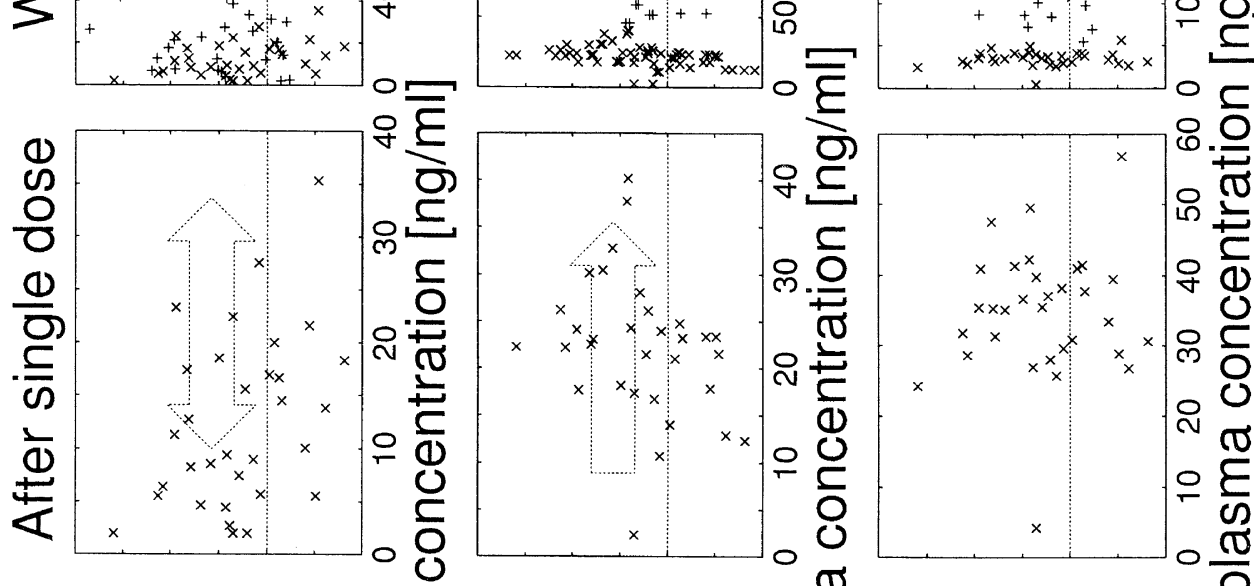

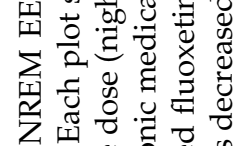

○

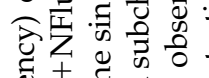

है

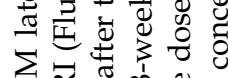

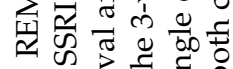

نे

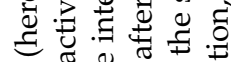

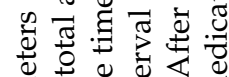

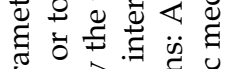

䒕

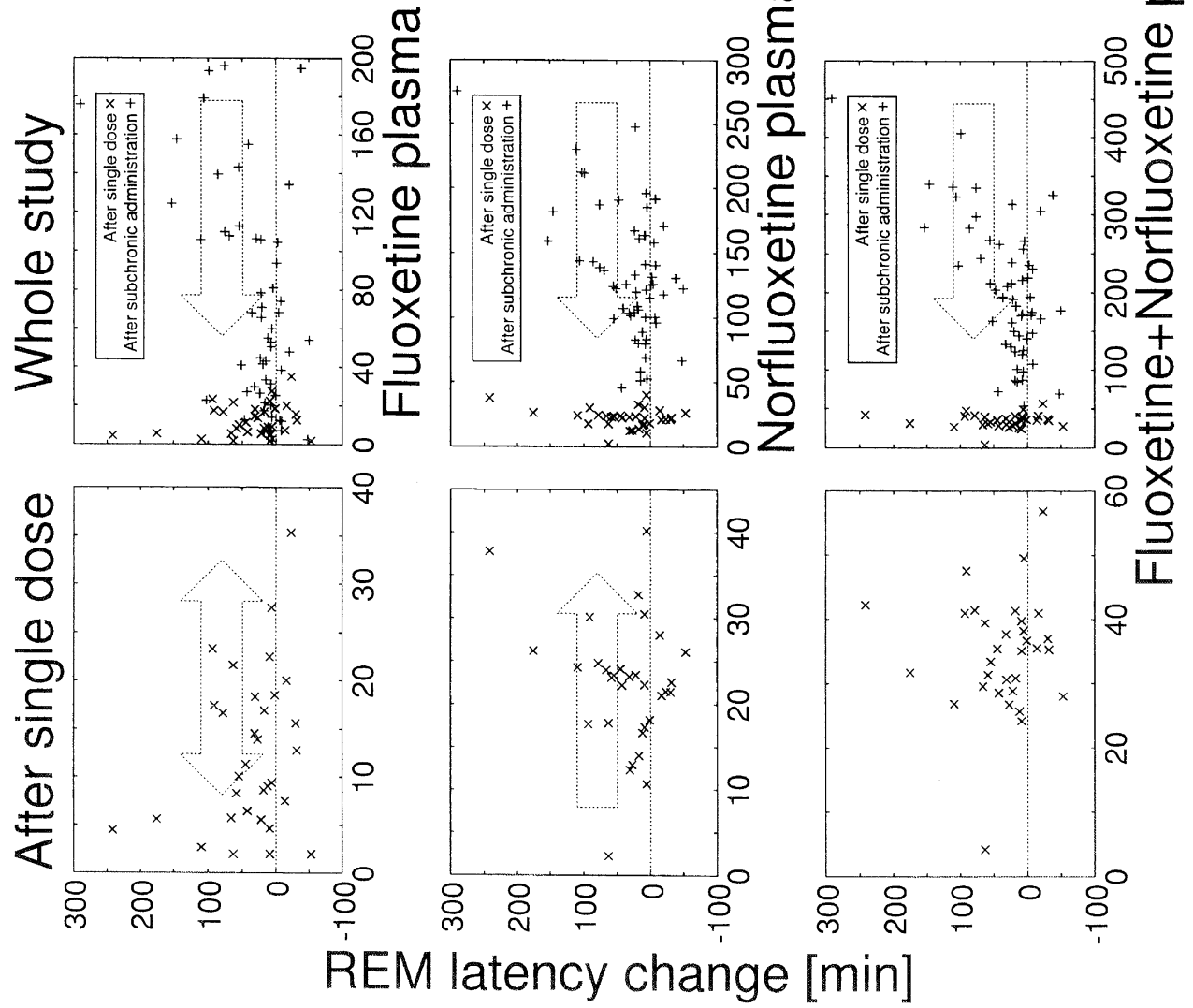

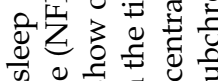

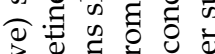

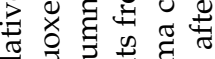

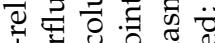

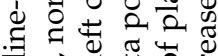

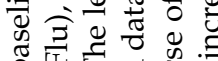

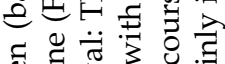

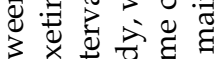

过苛志矛

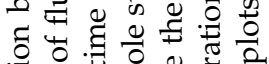

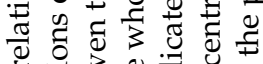

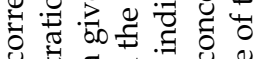

ठ

ङ

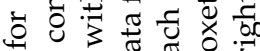

记

द्वे

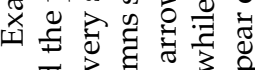

மं

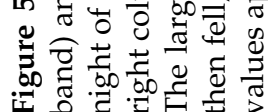


Table 2. Correlations with Fluoxetine/Norfluoxetine Plasma Concentrations

\begin{tabular}{|c|c|c|c|c|c|c|}
\hline & \multicolumn{3}{|c|}{$\begin{array}{c}\text { Correlation after Single Dose } \\
\text { (Nights 1-4) }\end{array}$} & \multicolumn{3}{|c|}{$\begin{array}{l}\text { Correlation after Subchronic } \\
\text { Administration (Nights 28-40) }\end{array}$} \\
\hline & Flu & NFlu & Flu + NFlu & Flu & NFlu & Flu + NFlu \\
\hline Sleep latency & - & - & - & - & - & - \\
\hline Total sleep time & - & - & - & - & - & - \\
\hline Sleep efficiency & - & - & - & - & - & - \\
\hline Wake \% SP & $\uparrow \uparrow$ & - & - & - & - & - \\
\hline Stage $1 \%$ SP & - & - & - & - & $\uparrow$ & - \\
\hline Stage $2 \% \mathrm{SP}$ & $\downarrow \downarrow \downarrow$ & - & $\downarrow \downarrow$ & $\downarrow$ & - & - \\
\hline SWS \% SP & - & - & - & $\uparrow$ & - & - \\
\hline REM \% SP & - & - & - & - & - & - \\
\hline REM latency & - & - & - & $\uparrow \uparrow \uparrow \uparrow \uparrow \uparrow$ & $\uparrow \uparrow \uparrow \uparrow \uparrow \uparrow$ & $\uparrow \uparrow \uparrow \uparrow \uparrow \uparrow \uparrow \uparrow$ \\
\hline REM lat.-wake & - & - & - & $\uparrow \uparrow \uparrow \uparrow$ & $\uparrow \uparrow \uparrow \uparrow$ & $\uparrow \uparrow \uparrow \uparrow \uparrow \uparrow \uparrow$ \\
\hline REM tot. eye mts. & - & - & - & - & - & - \\
\hline REM density & - & - & - & - & - & - \\
\hline Delta & - & - & - & - & - & - \\
\hline Theta & - & - & - & - & - & - \\
\hline Alpha & - & - & - & - & $\uparrow$ & $\uparrow$ \\
\hline Sigma & $\downarrow$ & - & - & $\uparrow \uparrow$ & $\uparrow \uparrow \uparrow$ & $\uparrow \uparrow \uparrow$ \\
\hline Beta1 & - & - & - & $\uparrow \uparrow$ & $\uparrow \uparrow \uparrow$ & 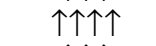 \\
\hline Beta2 & - & - & - & $\uparrow \uparrow$ & $\uparrow \uparrow$ & $\uparrow \uparrow \uparrow$ \\
\hline Delta ratio & - & - & - & - & - & - \\
\hline
\end{tabular}

$\uparrow$ positive, $\downarrow$ negative correlation; $p<.05 ; \uparrow \uparrow p<.01 ; \uparrow \uparrow \uparrow p<.001 ; \uparrow \uparrow \uparrow \uparrow p<10^{-4}$; $\uparrow \uparrow \uparrow \uparrow \uparrow ~ p<10^{-5} \ldots$

Correlations of the (baseline-relative) sleep and NREM EEG spectral parameters with the fluoxetine (Flu) and norfluoxetine (NFlu) plasma concentrations and with their sum, which corresponds to the total amount of SSRI. Correlations were determined independently for the time interval after the single dose of $60 \mathrm{mg}$ (nights 1-4) and for the time interval after 3-week administration of a daily $20 \mathrm{mg}$ dose (nights 28-40, cf. Fig. 5). Direction and number of the arrows indicate the direction and significance of each correlation.

- REM latency and spectral power increases across the alpha- to higher beta bands took longer to normalize after discontinuation and correlated with the sum of fluoxetine and norfluoxetine concentrations (total SSRI plasma concentration);

- The relative amount of REM sleep and the total rapid eye movement count showed a rebound effect, i.e., although there was a (tendential) reduction after both the single dose and subchronic dosing, they were significantly increased relative to baseline as soon as the drug plasma concentration was sufficiently decreased after discontinuation;

- The delta ratio remained elevated up to the end of the observation period (12 days after discontinuation) and showed no correlation with drug plasma levels.

\section{General Effect of Fluoxetine on the All-Night NREM Sleep EEG Spectrum}

At the end of the three-week medication interval, fluoxetine increased NREM sleep EEG spectral power in frequencies above $12 \mathrm{~Hz}$ relative to baseline, with a peak around $14 \mathrm{~Hz}$ and an increasing tendency towards the highest observed frequency $(32 \mathrm{~Hz})$. A slight decrease was observed in the delta band. This pattern is in line with previous reports of general activating effects of fluoxetine on the human sleep EEG (Saletu et al. 1991), including an increase in high- and a relative decrease in low-frequency activity, and a general alerting effect as reported by Blom et al. (1991). Despite the peak in the sigma band, no qualitative differences in drug response were observed between sigma and beta bands.

This spectral pattern is different from the SSRI effects consistently described in rats, namely a reduction of NREM sleep EEG spectral power in the $5-20 \mathrm{~Hz}$ range (Neckelmann et al. 1996 for citalopram; Bjorvatn et al. 1995 for zimelidine). Also, in awake depressed patients after four weeks of treatment with fluoxetine, Tarn et al. (1993) found a decrease in beta activity relative to baseline. Finally, Schlösser et al. (1998) reported a lack of significant NREM EEG spectral changes after four weeks of the SSRI paroxetine $(30 \mathrm{mg}$ ) in eight healthy subjects.

\section{Effects on Sleep Continuity Measures}

Similar to Kupfer et al. (1991) with the SSRI fluvoxamine, we found that sleep-disturbing effects of fluoxetine occurred in the initial medication phase, i.e., after the single dose of $60 \mathrm{mg}$, despite the fact that fluoxetine and norfluoxetine plasma levels were only slightly elevated by this dose. This means that sleep continuity could be described as sensitive to the change in plasma levels rather than to the absolute levels. The fact that sleep continuity was also significantly reduced after subchronic intake but recov- 
ered within 2-4 days indicates an additional influence of absolute plasma levels upon sleep continuity. The quick recovery may be explained by the effect of an increased homeostatic sleep pressure accumulated during the medication interval in which sleep continuity was reduced.

\section{Effects on REM Latency and NREM Sleep EEG Spectral Power}

Both REM latency and NREM sleep EEG spectral power effects covaried most clearly with the sum of fluoxetine and norfluoxetine plasma concentrations, both with regard to the mean time courses across the study (Figures 2 and 4) and with regard to the actual correlations between these parameters. This indicates that these effects are determined by the total plasma concentration of active SSRI rather than by fluoxetine or norfluoxetine alone, and do not show habituation within the sensitivity of the current study. Changes in REM latency excluding intervening wake periods were nearly equal to and only slightly less significant than those including wake periods, indicating that the observed changes in REM latency are not due to the overall decrease of sleep continuity (increase in wake \% SP).

Due to the apparent dependence of spectral power effects upon total SSRI plasma concentration, an $E_{\max }$ pharmacodynamic model could be applied. In all examined spectral bands (sigma, beta1, and beta2), the standard deviation of the $E_{\max }$ parameter across subjects, which measures the actual influence of plasma concentration on spectral power, was nearly as large as the average $E_{\max }$. This indicates that a large interindividual variability is not only present in the pharmacokinetics of fluoxetine but also in the effect of a given plasma concentration on NREM sleep EEG spectral power.

An unexpected finding was a slight increase in spectral power in the beta frequency ranges at night 34 , i.e. six days after discontinuation, despite the continuous decline of plasma drug levels. It is tempting to propose a link between this high-frequency increase, the REM rebound observed at night 32 and the delta ratio increase observed at night 34 . However, further studies are needed to validate this observation.

\section{Habituation and Rebound Effects on REM Sleep}

Both the REM fraction of the sleep period and the total number of rapid eye movements (total REM activity) showed a habituation and rebound pattern of changes. Since REM density only showed a slight, not significant increase on day 28 , the change in total REM eye movements appears to be mostly governed by the increase in REM percentage, i.e., by an aspect of REM generation. REM latency but not REM density also clearly covaried with the total SSRI plasma level. This suggests that tonic and phasic REM activity are modified in different ways by fluoxetine treatment, at least in healthy controls.

It is tempting to assume a correspondence between the observed rebound effects and possible SSRI discontinuation symptoms. However, there are no studies characterizing the time course of such symptoms for fluoxetine; in fact, some studies have failed to find a significant increase of complaints after discontinuation at all (Zajecka et al. 1998; Rosenbaum et al. 1998). Black et al. (2000) concluded in a review of reports for shorter-halflife SSRIs that discontinuation symptoms begin 1-3 days after discontinuation and last for more than a week in half of the cases, which could correspond to the significant rebound effects we observed between four and 12 days after discontinuation.

\section{Prolonged Increase in Delta Ratio}

Kupfer et al. (1989) observed no change in whole-night delta wave count in depressives after clomipramine, but a rearrangement of delta activity towards the first NREM episode, as indicated by an increased delta sleep ratio. In the current study, delta ratio was slightly elevated by the initial dose and clearly elevated after the three-week medication interval. At the same time, it showed no correlation with drug plasma concentrations. This indicates that the delta ratio change is caused by secondary, adaptive processes and probably does not occur consistently across subjects. A low delta ratio has been described as a predictor for recurrence of depression (Kupfer et al. 1990), and in longitudinal studies an increase in delta ratio has been found to be associated with remission (Buysse et al. 1997), thus pointing at both 'state' and 'trait' relevance of this parameter. Since variations in depressive vulnerability and mood are continuous and certainly present in healthy subjects, one could hypothesize that the observed elevation in delta ratio in healthy controls could be due to such variations within our subjects.

\section{Implications for Clinical Application}

A daily intake of $40 \mathrm{mg}$ of fluoxetine for three weeks resulted in clear effects in sleep and NREM EEG spectral power variables, including REM latency and delta ratio, which have previously been linked to the effect of antidepressants. Whether a maintenance treatment could use other than daily application schemes, e.g. once every few days, depends upon which of these variables will represent a valid correlate of the antidepressant effect. The correlation of REM latency and spectral power effects with SSRI plasma concentration suggests that the plasma concentration would have to remain high during maintenance therapy. The REM fraction of the sleep period habituated, thereby suggesting that REM fraction is probably not indicative of the antidepressant 
effect. Finally, the delta sleep ratio did not correlate with drug plasma concentrations but remained elevated after treatment. Thus, if delta ratio increase was a correlate of the antidepressive effect, fluoxetine doses separated by some days might be effective in maintenance therapy after an initial daily phase.

\section{ACKNOWLEDGMENTS}

This study was supported by a grant from Eli Lilly \& Co, Germany. The authors wish to thank the anonymous referees for their constructive criticism and comments.

\section{REFERENCES}

Achermann P, Dijk DJ, Brunner DP, Borbély AA (1993): A model of human sleep homeostasis based on EEG slowwave activity: Quantitative comparison of data and simulations. Brain Res Bull 31:97-113

Armitage R, Yonkers KA, Cole D, Rush AJ (1997): A multicenter, double-blind comparison of the effects of nefazodone and fluoxetine on sleep architecture and quality of sleep in depressed outpatients. J Clin Psychopharmacol 17:161-168

Bellissant E, Sebille V, Paintaud G (1998): Methodological issues in pharmacokinetic-pharmacodynamic modelling. Clin Pharmacokinet 35:151-166

Berger M, Riemann D (1993): REM sleep in depression. J Sleep Res 2:211-223

Berger M, Vollmann J, Hohagen F, König A, Lohner H, Voderholzer U, Riemann D (1997): Sleep deprivation combined with consecutive sleep phase advance as a fast-acting therapy in depression: An open pilot trial in medicated and unmedicated patients. Am J Psychiatry 154:870-872

Bjorvatn B, Bjorkum AA, Neckelmann D, Ursin R (1995): Sleep/waking and EEG power spectrum effects of a nonselective serotonin (5-HT) antagonist and a selective 5 -HT reuptake inhibitor given alone and in combination. Sleep 18:451-462

Black K, Shea C, Dursun S, Kutcher S (2000): Selective serotonin reuptake inhibitor discontinuation syndrome: Proposed diagnostic criteria. J Psychiatry Neurosci 25:255-261

Blom MW, Sommers DK, Bartel PR, van der Meyden $\mathrm{CH}$, Becker PJ (1991): A comparison of psychomotor and EEG effects of desipramine, clomipramine and fluoxetine. Med Sci Res 19:751-752

Borbély AA, Achermann P, Trachsel L, Tobler I (1988): Sleep homeostasis in humans and rats. In Lydic R, Biebuyck JF (eds), Clinical Physiology of Sleep. Bethesda, MD, Americal Physiological Society, pp 191-198

Buysse DJ, Frank E, Lowe KK, Cherry CR, Kupfer DJ (1997): Electroencephalographic sleep correlates of episode and vulnerability to recurrence in depression. Biol Psychiatry 41:406-418

Buysse DJ, Kupfer DJ, Cherry C, Stapf D, Frank E (1999): Effects of prior fluoxetine treatment on EEG sleep in women with recurrent depression. Neuropsychopharmacology 21:258-267

DeVane CL (1992): Pharmacokinetics of the selective serotonin reuptake inhibitors. J Clin Psychiatry 53:13-20

Ehlers CL, Havstad JW, Kupfer DJ (1996): Estimation of the time course of slow-wave sleep over the night in depressed patients: Effects of clomipramine and clinical response. Biol Psychiatry 39:171-181

Feige B, Voderholzer U, Riemann D, Hohagen F, Berger M (1999): Independent sleep EEG slow-wave and spindle band dynamics associated with 4 weeks of continuous application of short-halflife hypnotics in healthy subjects. Clin Neurophysiol 110:1965-1974

Fischer W, Streubel FR, Heydenreich F, Rabending G (1986): Quantitative EEG analysis of a single dose of psychotropic drugs in healthy probands. Psychiatrie Neurologie Medizinische Psychologie 38:450-455

Gillin JC, Rapaport M, Erman MK, Winokur A, Albala BJ (1997): A comparison of nefazodone and fluoxetine on mood and on objective, subjective, and clinician-rated measures of sleep in depressed patients: A doubleblind, 8-week clinical trial. J Clin Psychiatry 58:185-192

Haddad P (1997): Newer antidepressants and the discontinuation syndrome. J Clin Psychiatry 58:17-22

Hyttel J (1994): Pharmacological characterization of selective serotonin reuptake inhibitors (SSRIs). Int Clin Psychopharmacol 9:19-26

Kupfer DJ, Ehlers CL, Frank E, Grochocinski VJ, McEachran AB, Buhari A (1994): Persistent effects of antidepressants: EEG sleep studies in depressed patients during maintenance treatment. Biol Psychiatry 35:781-793

Kupfer DJ, Ehlers CL, Pollock BG, Nathan S, Perel JM (1989): Clomipramine and EEG sleep in depression. Psychiatry Res 30:165-180

Kupfer DJ, Foster FG, Reich L, Thompson SK, Weiss B (1976): EEG sleep changes as predictors in depression. Am J Psychiatry 133:622-626

Kupfer DJ, Frank E, McEachran AB, Grochocinski VJ (1990): Delta sleep ratio. A biological correlate of early recurrence in unipolar affective disorder. Arch Gen Psychiatry 47:1100-1105

Kupfer DJ, Perel JM, Pollock BG, Nathan RS, Grochocinski VJ, Wilson MJ, McEachran AB (1991): Fluvoxamine versus desipramine: Comparative polysomnographic effects. Biol Psychiatry 29:23-40

Lejoyeux M, Adés J (1997): Antidepressant discontinuation: A review of the literature. J Clin Psychiatry 58:11-16

Malhotra S, Santosh PJ (1996): Loading dose imipraminenew approach to pharmacotherapy of melancholic depression. J Psychiatr Res 30:51-58

Neckelmann D, Bjorkum AA, Bjorvatn B, Ursin R (1996): Sleep and EEG power spectrum effects of the 5-HT1A antagonist NAN-190 alone and in combination with citalopram. Behav Brain Res 75:159-168

Patat A, Troy S, Burke J, Trocherie S, Danjou P, Le CF, Allain H, Gandon JM (1998): Absolute bioavailability and electroencephalographic effects of conventional and extended-release formulations of venlafaxine in healthy subjects. J Clin Pharmacol 38:256-267

Pollock BG, Perel JM, Nathan S, Kupfer DJ (1989): Acute 
antidepressant effect following pulse loading with intravenous and oral clomipramine. Arch Gen Psychiatry 46:29-35

Quitkin FM, Rabkin JD, Markowitz JM, Stewart JW, McGrath PJ, Harrison W (1987): Use of pattern analysis to identify true drug response. A replication. Arch Gen Psychiatry 44:259-264

Quitkin FM, Rabkin JG, Ross D, Stewart JW (1984): Identification of true drug response to antidepressants. Use of pattern analysis. Arch Gen Psychiatry 41:782-786

Rechtschaffen A, Kales A (1968): A manual of standardized terminology, techniques, and scoring system for sleep stages of human subjects. (Publication 204). Washington, DC, National Institutes of Health

Riemann D, König A, Hohagen F, Kiemen A, Voderholzer U, Backhaus J, Bunz J, Wesiack B, Hermle L, Berger M (1999): How to preserve the antidepressive effect of sleep deprivation: A comparison of sleep phase advance and sleep phase delay. Eur Arch Psychiatry Clin Neurosci 249:231-237

Röschke J, Kogel P, Schlosser R, Wagner P, Mann K, Rossbach W, Benkert O (1997): Analysis of sleep EEG microstructure in subchronic paroxetine treatment of healthy subjects. Psychopharmacology 132:44-49

Rosenbaum JF, Fava M, Hoog SL, Ascroft RC, Krebs WB (1998): Selective serotonin reuptake inhibitor discontinuation syndrome: A randomized clinical trial. Biol Psychiatry 44:77-87

Rush AJ, Armitage R, Gillin JC, Yonkers KA, Winokur A, Moldofsky H, Vogel GW, Kaplita SB, Fleming JB, Montplaisir J, Erman MK, Albala BJ, McQuade RD (1998): Comparative effects of nefazodone and fluoxetine on sleep in outpatients with major depressive disorder. Biol Psychiatry 44:3-14

Saletu B, Frey R, Krupka M, Anderer P, Grünberger J, See WR (1991): Sleep laboratory studies on the single-dose effects of serotonin reuptake inhibitors paroxetine and fluoxetine on human sleep and awakening qualities. Sleep 14:439-447

Saletu B, Grünberger J (1985): Classification and determination of cerebral bioavailability of fluoxetine: Pharmacokinetic, pharmaco-EEG, and psychometric analyses. J Clin Psychiatry 46:45-52

Schatzberg AF, Haddad P, Kaplan EM, Lejoyeux M, Rosenbaum JF, Young AH, Zajecka J (1997): Serotonin reuptake inhibitor discontinuation syndrome. A hypothetical definition. J Clin Psychiatry 58:5-10

Schlösser R, Röschke J, Rossbach W, Benkert O (1998): Conventional and spectral power analysis of all-night sleep EEG after subchronic treatment with paroxetine in healthy male volunteers. Eur Neuropsychopharmacol 8:273-278

Schmidt ME, Fava M, Robinson JM, Judge R (2000): The efficacy and safety of a new enteric-coated formulation of fluoxetine given once weekly during the continuation treatment of major depressive disorder. J Clin Psychiatry 61:851-857
Sonntag A, Rothe B, Guldner J, Yassouridis A, Holsboer F, Steiger A (1996): Trimipramine and imipramine exert different effects on the sleep EEG and on nocturnal hormone secretion during treatment of major depression. Depression 4:1-13

Spanier C, Frank E, McEachran AB, Grochocinski VJ, Kupfer DJ (1996): The prophylaxis of depressive episodes in recurrent depression following discontinuation of drug therapy: Integrating psychological and biological factors. Psychol Med 26:461-475

Stassen HH, Angst J (1998): Delayed onset of action of antidepressants - fact or fiction? CNS Drugs 9:177-184

Tarn M, Edwards JG, Sedgwick EM (1993): Fluoxetine, amitriptyline and the electroencephalogram. J Affect Dis 29:7-10

Trivedi M, Rush AJ, Armitage R, Gullion CM, Grannemann BD, Orsulak PJ, Roffwarg HP (1999): Effects of fluoxetine on the polysomnogram in outpatients with major depression. Neuropsychopharmacology 20:447-459

Vasar V, Appelberg B, Rimon R, Selvaratnam J (1994): The effect of fluoxetine on sleep: A longitudinal, doubleblind polysomnographic study of healthy volunteers. Int Clin Psychopharmacol 9:203-206

Viguera AC, Baldessarini RJ, Friedberg J (1998): Discontinuing antidepressant treatment in major depression. Harvard Rev Psychiatry 5:293-306

Vogel GW (1983): Evidence for REM sleep deprivation as the mechanism of action of antidepressant drugs. Prog Neuropsychopharmacol Biol Psychiatry 7:343-349

Vogel GW, Buffenstein A, Minter K, Hennessey A (1990): Drug effects on REM sleep and on endogenous depression. Neurosci Biobehav Rev 14:49-63

Vogel GW, Cohen J, Mullis D, Kensler TT, Kaplita SB (1998): Nefazodone and REM sleep: How do antidepressant drugs decrease REM sleep? Sleep 21:70-77

Vogel GW, Thurmond AJ, Gibbons P, Sloan K, Walker M (1975): REM sleep reduction effects on depression syndromes. Arch Gen Psychiatry 32:765-777

Vollmann J, Berger M (1993): Sleep deprivation with consecutive sleep-phase advance therapy in patients with major depression: A pilot study. Biol Psychiatry 33:54-57

Wiegand M, Berger M (1989): Action of trimipramine on sleep and pituitary hormone secretion. Drugs 38(Suppl 1):35-50

Yamadera H, Ferber G, Matejcek M, Pokorny R (1987): Quantitative pharmaco-electroencephalographic differentiation between the CNS effects of bromocriptine and imipramine, drugs with qualitatively different antidepressant properties. Pharmacopsychiatry 20:54-59

Zajecka J, Fawcett J, Amsterdam J, Quitkin FM, Reimherr F, Rosenbaum JF, Michelson D, Beasley C (1998): Safety of abrupt discontinuation of fluoxetine: A randomized, placebo-controlled study. J Clin Psychopharmacol 18:193-197 